Adding, therefore, the cross-sections (1) and (4), and also (3) and (5), we obtain finally:

$$
\begin{gathered}
\mathrm{d}^{(2)} \text { Compton }=\frac{\alpha^{3} \mathbf{K}^{2} \mathrm{~d} \Omega_{K^{\prime}}}{3 \pi K_{0}{ }^{4}}\left[2(1-\cos \theta)\left(1+\cos ^{2} \theta\right) \times\right. \\
\log \frac{2 \mathbf{K}^{2}(1-\cos \theta)}{e K_{0}^{2}}+6 y\left(1+\cos \theta-\frac{1}{3} \cos ^{3} \theta+\right. \\
\left.\left.\cos ^{2} \theta\right)+\frac{8}{3}-\frac{13 \cos \theta}{2}+\frac{8 \cos ^{2} \theta}{3}+\frac{5 \cos ^{3} \theta}{6}\right](A) \\
\mathrm{d}^{(2)} \Phi_{\text {Bremss. }}=\frac{4 \alpha^{4} Z^{2} \mathrm{~d} \Omega_{q} \mathrm{~d} K}{9 \pi^{2}{ }_{1} \mathbf{K} K_{0}{ }^{2}} \times\left|\frac{\mathbf{q}}{\mathbf{p}}\right| \times \\
\left(4 \log \frac{4 \mathbf{q}^{2}}{k_{0}{ }^{2}}-\frac{4 \mathrm{l}}{5}\right) .
\end{gathered}
$$

These expressions are completely free from the infra-red divergences. The ultra-violet divergences have been already removed by re-normalization methods. Details of this work will be published shortly.

I am indebted to Prof. R. C. Majumdar for suggesting this problem and giving me guidance and encouragement throughout the work.

Department of Physics,

A. N. Mitra

University of Delhi.

Dec. 1.

\section{- Surface Tension and Melting Point}

WEALE ${ }^{1}$ has directed attention to an earlier formula, due to Schytil, according to which the surface tension of a liquid is proportional to $T_{m} V^{-2 / 3}, T_{m}$ being the melting point and $V$ the molar volume. Since the number of molecules in a unit area of the surface is $(V / N)^{-2 / 3}$, the above relation can be interpreted to mean that the surface energy per unit area, and the thermal energy of the molecules in that area, are proportional to each other at the melting point. Numerical calculations show that for most liquids the two are almost equal, if we consider the translational part of the thermal energy only. Thus $\sigma_{m}$, the surface tension at the melting point, is given by:

$$
\sigma_{m}=3(N / V)^{2 / 3} k T_{m},
$$

$k$ being the Boltzmann constant.

A number of unassociated organic liquids obey the above relation. Small discrepancies can often be explained by the fact that the distance, $d$, between the two adjacent surface layers is not given exactly by $(V / N)^{1 / 8}$, so that the number of molecules per unit surface is slightly different from that assumed above. The accurate expression for $\sigma_{m}$ is then :

$$
\sigma_{m}=3(N / V) d k T_{m}
$$

Equation (2) gives an accurate value of $\sigma_{m}$ in the case of benzene, where $d$ is known from $\mathrm{X}$-ray diffraction studies ${ }^{2}$ to be $3.7 \mathrm{~A}$. If there is any association of the molecules, there is a decrease in their number in the surface layer, and the righthand side of equations (1) and (2) must be multiplied by an association factor. As an example, the association factor to be used in equation (1) for explaining the observed surface tension of acetic acid is $\frac{1}{2}$, thus showing, in agreement with the general view, that two molecules have become associated to form a single unit.
The ratio $3 k T_{m} / \sigma_{m}$ gives the area of the surface covered by a single molecule. In the case of those organic compounds in which the hydrocarbon chain is perpendicular to the surface, the cross-section of a single chain can be obtained on multiplying this area by the appropriate association factor. This has been calculated for a few esters and acids (see table), the experimental results for $\sigma_{m}$ having been extrapolated roughly from the values given in the "International Critical Tables". The association factor has been taken to be 1 in the case of esters and $\frac{1}{2}$ for the acids, as is generally accepted. The calculated crosssection is in fair agreement with that obtained from $\mathrm{X}$-ray diffraction and from monomolecular layers.

\begin{tabular}{|l|c|c|c|}
\multicolumn{1}{|c}{ Cross-SECTION OF A HYDROCARBON CHAIN } \\
\hline \multicolumn{1}{|c|}{ Substance } & $\begin{array}{c}\sigma_{m} \\
\text { (dynes/cm.) }\end{array}$ & $\begin{array}{c}T_{m} \\
\left({ }^{\circ} \mathbf{K} .\right)\end{array}$ & $\begin{array}{c}\text { Cross-section of } \\
\text { hydrocarbon chain } \\
\text { (sq. A.) }\end{array}$ \\
\hline Ethyl formate & $36 \cdot 6$ & 193 & $21 \cdot 8$ \\
Ethyl acetate & $37 \cdot 7$ & 189 & $20 \cdot 5$ \\
Methyl propionate & $36 \cdot 7$ & 186 & $20 \cdot 7$ \\
Ethyl propionate & 35 & 200 & $23 \cdot 5$ \\
Isobutyl acetate & 34 & 173 & 21 \\
Ethyl isovalerate & 34 & 174 & 21 \\
Acetic acid & $28 \cdot 2$ & 290 & $21 \cdot 1$ \\
n-Butyric acid & $29 \cdot 6$ & 265 & $18 \cdot 5$ \\
Undecylenic acid & $30 \cdot 6$ & 298 & 20 \\
Oleic acid & $32 \cdot 5$ & 287 & $18 \cdot 2$ \\
\hline
\end{tabular}

Equation (1) gives too high a value of $\sigma_{m}$ for the alkali halides, so that we have to assume some degree of association, a conclusion also necessitated by the abnormal values of their Eötvös constants. We may, however, avoid this assumption and obtain the experimental value of $\sigma_{m}$ by using equation (2), with the values of $d$ obtained from the spacings of the (111) and (110) planes for the sodium chloride and the cæsium chloride types of lattices respectively, and after making allowance for the thermal expansion from room temperature to the melting point. In the sodium chloride lattice, the (111) planes contain all the atoms of one kind only, so that in order to keep complete molecules in the surface layer we may have to make an additional postulate that half the alkali atoms from one plane have exchanged sites, in the liquid state, with the halogens of the adjacent planes.

In the case of the fused metals of the cubic system, the values of $\sigma_{m}$ derived from equation (1) are found to be slightly but consistently smaller than the experimental values; equation (2), with $d$ derived from standard crystallographic planes, shows no improvement. The difference between the experimental and the calculated values can, perhaps, be accounted for on the basis that it represents the kinetic energy of the free electrons, which can only be possible if the density of the electrons in the surface layer is considerably smaller than that in the bulk of the liquid metal.

The above results show that just as the critical temperature is characterized by a zero value of the surface tension, the melting point may be defined as the temperature where the thermal energy of the translation of the molecules of a given surface becomes equal to its surface free energy. So long as the former is smaller, the surface preserves its characteristic solid form.

Department of Physics,

\section{Bisheshwar Dayal}

Banaras Hindu University. Oct. 19.

1 Weale, Nature, 168, 343 (1951).

"Glasstone, "Text-Book of Physical Chemistry", 509 (Macmillan and Van Nostrand Co., 1946). 